\title{
The histone demethylase PHF8 promotes prostate cancer cell growth by activating the oncomiR miR-I25b
}

This article was published in the following Dove Press journal:

OncoTargets and Therapy

10 August 2015

Number of times this article has been viewed

\section{Qiang Ma* \\ Zhuo Chen* \\ Guojin Jia \\ Xueqiang Lu \\ Xuefeng Xie \\ Wei Jin}

Department of Urology, Jinshan Hospital, Fudan University, Shanghai, People's Republic of China

*These authors contributed equally to this work
Correspondence: Wei Jin

Department of Urology, Jinshan Hospital, Fudan University, No 1508 Longhang Road, Shanghai 201508, People's Republic of China

Tel +86 21 34189990

Email jinwei_ch@I63.com
Aims: Prostate cancer (PCa) is the most frequently diagnosed malignancy in men. However, the underlying mechanism is not fully understood. In this study, we aim to research the molecular mechanisms underlying the initiation and progression of $\mathrm{PCa}$.

Results: Plant homeodomain finger protein 8 (PHF8) is upregulated in human PCa tissues and cell lines. PHF8 knockdown attenuates growth and cellular transformation of PCa cells. PHF8 depletion induces PCa cell apoptosis by activating proapoptotic proteins and inactivating antiapoptotic proteins. Furthermore, miR-125b is a target of PHF8, and miR-125b seems to be essential for the hyper proliferation of PCa cells in the presence of PHF8.

Conclusion: In conclusion, we identify the histone demethylase PHF8 as an oncogenic protein in human PCa. These findings indicate PHF8 as a potential candidate for clinical intervention.

Keywords: PHF8, prostate cancer, apoptosis, miR-125b

\section{Introduction}

Prostate cancer ( $\mathrm{PCa})$ is the most frequently diagnosed malignancy in men and the second leading cause of cancer deaths among men in Western countries. ${ }^{1}$ There is still an urgent need for appropriate diagnostic and prognostic markers for $\mathrm{PCa}$, in addition to the established serum protease prostate-specific antigen. Although the involvement of certain genes and chromosomal aberrations in prostate carcinogenesis has been suggested, ${ }^{2,3}$ the molecular mechanisms underlying the initiation and progression of $\mathrm{PCa}$ are poorly understood.

Plant homeodomain finger protein 8 (PHF8) is a JmjC domain-containing protein and erases repressive histone marks, including H4K20me1 and H3K9me1/2. ${ }^{4-6} \mathrm{PHF} 8$ binds to $\mathrm{H} 3 \mathrm{~K} 4 \mathrm{me} 3$, an active histone mark usually located at transcription start sites, through its plant homeodomain, and is thus recruited and enriched at promoters of target genes. ${ }^{7,8}$ Chromatin immunoprecipitation-sequencing data from HeLa cells show that approximately $72 \%$ of PHF8-binding sites are at promoters. ${ }^{5}$ Also, PHF8 regulates the cell cycle biological processes via removing H4K20me1 from the promoters of certain E2F1-regulated genes. ${ }^{4}$ Interestingly, altering PHF8 levels in HeLa cells affects H4K20me1 methylation only in late G2/M and early G1 stages of the cell cycle, not globally. ${ }^{4} \mathrm{PHF} 8$ also binds to rRNA gene promoters and demethylates H3K9me2/1 to activate rRNA synthesis, ${ }^{6,9}$ functioning as an activator. ${ }^{10}$ However, the role of PHF8 in human diseases, especially carcinogenesis, is largely unknown.

MicroRNAs have been widely considered in the initiation and development of cancer. Both local and circulating microRNAs have been reported to participate in human PCa. ${ }^{11-14}$ For instance, the oncomiR miR-125b has been demonstrated to promote 
the development of $\mathrm{PCa}$, and the underlying mechanism has been widely investigated. ${ }^{13,15-19}$ Nevertheless, how miR-125b is regulated in $\mathrm{PCa}$ remains elusive.

In the present work, we find that PHF8 is overexpressed in human PCa tissues and cell lines. The results show that PHF8 regulates PCa cell growth, cellular transformation, and survival by regulating the oncomiR miR-125b.

\section{Materials and methods}

\section{Patient identification and pathology evaluation}

Radical prostatectomy specimens were available from 89 patients treated at the Jinshan Hospital, Fudan University (Shanghai) between 2001 and 2009. Tumors were diagnosed and classified according to the Gleason system. The 19 adjacent normal tissues were obtained from PCa patients undergoing surgery. A written form of informed consent was obtained from all the patients, and the study was approved by the Clinical Research Ethics Committee of Fudan University.

\section{Immunohistochemical assay}

Tissues were fixed with $4 \%$ neutral formalin. Cancer or adjacent tissue sections were subjected to immunohistochemical staining with anti-PHF8 antibody. Paraffin sections were subjected to high-temperature antigen retrieval, 3 minutes in a pressure cooker in $0.01 \mathrm{M}$ citrate buffer $\mathrm{pH}$ 6.0. Slides were treated with $3 \% \mathrm{H}_{2} \mathrm{O}_{2}$ for 15 minutes, blocked in 5\% normal horse serum in phosphate-buffered saline for 20 minutes, and stained with anti-PHF8 antibody in 5\% normal goat serum overnight at $4^{\circ} \mathrm{C}$. Secondary antibody was used according to VECTASTAIN ABC ${ }^{\circledR}$ kits, followed by DAB staining. The areas of total prostate tumors and PHF8-positive areas were quantified using ImageJ. The average percentage of PHF8-positive area is $31 \%$. This median value was used to cut off the subgroups of all immunohistochemical variables in our data. The patients were then divided into two groups: PHF8 high expression group ( $\geq 31 \%$ PHF8-positive/total tissue cores, $\mathrm{n}=41)$ and PHF 8 low expression group $(<31 \%$ PHF8-positive/total tissue cores, $\mathrm{n}=48$ ).

\section{Western blot}

PCa tissues and cells were lysed with cell lysis buffer (Beyotime Institute of Technology, Shanghai, People's Republic of China). Total proteins were applied to SDS-polyacrylamide gel. After electrophoresis, the proteins were transferred to poly(vinylidene fluoride) membranes, followed by blocking in the buffer containing 5\% fat-free dry milk. The membranes were then probed with indicated antibodies overnight, and then washed and incubated with HRP-conjugated secondary antibodies (Zhongsan Jinqiao Biotechnology, Beijing, People's Republic of China) for 1.5 hours, and finally, visualized using Chemiluminescent ECL reagent (Vigorous Biotechnology, Beijing, People's Republic of China). The following antibodies were used: anti-GAPDH (Cell Signaling Technology, Beverly, MA, USA), anti-PHF8 (Cell Signaling Technology), anti-poly(ADP-ribose) polymerase (Abcam, Cambridge, UK), anti-caspase 3 (Abcam), anti-Bax (Abcam), anti-Bcl-2 (Santa Cruz Biotechnology Inc., Dallas, TX, USA), and anti-p21 (Santa Cruz).

\section{Quantitative real-time PCR}

Total RNA was extracted from cells or tissues with TRIzol ${ }^{\circledR}$, and cDNA was synthesized from $1 \mu \mathrm{g}$ of RNA with one-Step RT-PCR Kit. q-PCR was performed with the SYBR Green detection method on an ABI-7500 RT-PCR system (Thermo Fisher Scientific, Waltham, MA, USA). GAPDH was used as housekeeping gene. The primers used are listed in Table S1.

\section{Cell lines and cell culture}

The human prostate carcinoma cell lines LNCaP, DU145, and PC3 were obtained from the American Type Culture Collection ([ATCC], Manassas, VA, USA), PTN2 from SigmaAldrich Co. (St Louis, MO, USA), and BPH-1 from YRGenge (Changsha, Hunan, the People's Republic of China), and were grown in RPMI 1640 (Thermo Fisher Scientific) supplemented with 10\% FBS (Thermo Fisher Scientific).

\section{Package of retrovirus and transduction}

Control sh-RNA and specific sh-RNAs targeting PHF8 were purchased from Thermo Fisher Scientific, and the corresponding sequences were cloned into the pSIREN-RetroQ plasmid (Addgene, Cambridge, MA, UK) for retrovirus production with $293 \mathrm{~T}$ cells. The targeting sequences are listed in Table S2. For transduction, 293T cells were incubated with virus-containing supernatant in the presence of $8 \mathrm{mg} /$ $\mathrm{mL}$ polybrene. After 48 hours, infected cells were selected with puromycin $(2 \mathrm{mg} / \mathrm{mL})$. Then, the clones were picked and cultured for further experiment.

For transfection, the locked nucleic acid (LNA)-antimiR125b or LNA-control (Exiqon A/S, Skelstedet, Vedbaek, Denmark) was delivered at a final concentration of $50 \mathrm{nM}$ using Lipofectamine ${ }^{\circledR} 2000$ reagent (Thermo Fisher Scientific).

\section{Cell proliferation assay}

Cell proliferation rate was tested with MTT assay. The MTT assay was performed using MTT Cell Proliferation and 
Cytotoxicity Assay Kit (R\&D Systems, Inc., Minneapolis, MN, USA) according to the manufacturer's protocol.

\section{Soft sugar colony formation assay}

PCa cells were suspended in $1.5 \mathrm{~mL}$ complete medium supplemented with $0.45 \%$ low melting point agarose (Thermo Fisher Scientific). The cells were placed in $35 \mathrm{~mm}$ tissue culture plates containing $1.5 \mathrm{~mL}$ complete medium and agarose $(0.75 \%)$ on the bottom layer. The plates were incubated at $37^{\circ} \mathrm{C}$ with $5 \% \mathrm{CO}_{2}$ for 2 weeks. Cell colonies were stained with $0.005 \%$ crystal violet and analyzed using a microscope.

\section{In vivo tumor growth experiment}

Xenograft mice experiments were performed as described previously. ${ }^{20}$ In brief, equal numbers of PHF8 cells expressing either control or PHF8 knockdown vectors were injected subcutaneously, within 30 minutes of harvesting, over the right and left flanks in male nu/nu mice between 4 weeks and 6 weeks of age. Tumor growth was monitored using calipers. The mice were killed, and the tumors were weighted 3 weeks after inoculation. $\mathrm{N}=10$ in each group. The experimental protocol used in this study has been reviewed and approved by the Jinshan Hospital affiliated to Fudan University -Institutional Animal Care and Use Committee on their ethical procedures and scientific care.

\section{Apoptotic assays}

Cells were washed twice with cold PBS, and then resuspended in $1 \times$ binding buffer (BD Biosciences, San Jose, CA, USA) at a concentration of $1 \times 10^{6}$ cells $/ \mathrm{mL}$. Then, $100 \mathrm{~mL}$ of the solution $\left(1 \times 10^{5}\right.$ cells $)$ was transferred to a $5 \mathrm{~mL}$ culture tube and stained with $5 \mathrm{~mL}$ each of allophycocyaninannexin $\mathrm{V}$ (BD Biosciences) and $50 \mathrm{mg} / \mathrm{mL}$ propidium iodide (Thermo Fisher Scientific). The cells were gently mixed and incubated at room temperature for 15 minutes. For assessment of apoptosis, $400 \mathrm{~mL}$ of $1 \times$ binding buffer was then added to each tube and the samples were analyzed by flow cytometry using a LSRII instrument (Becton, Dickinson and Company, Franklin Lakes, NJ, USA).

The induction of apoptosis was also monitored by terminal deoxynucleotidyl transferase-mediated dUTP nick end labeling (TUNEL) method. The TUNEL assay was performed according to the guidelines recommended by the TUNEL Apoptosis Kit (R\&D Systems, Inc.).

\section{Luciferase assay}

To generate the luciferase reporter vectors, an internal ribosome entry site was amplified from pMSCV-PIG and cloned into the BglII site of pGL3-Basic (Promega Corporation, Fitchburg, WI, USA). miR-125b promoter fragments ${ }^{21}$ were amplified from human genomic DNA and cloned into the XhoI site. Twenty-four hours before transfection, $7 \times 10^{4}$ cells were plated per well in a 24 -well plate. pGL3 constructs (100 ng) plus $1 \mathrm{ng}$ of the Renilla luciferase plasmid phRL-SV40 (Promega) were transfected using FuGENE 6 (Hoffman-La Roche Ltd., Basel, Switzerland). Twenty-four hours after transfection, luciferase assays were performed using the dual luciferase reporter assay system (Promega). Firefly luciferase activity was normalized to Renilla PHF8mediated transactivation of miR-125b luciferase activity for each transfected well. Full-length human PHF8 was cloned into the pcDNA4 vector for expression in mammalian cells. For each experimental trial, wells were transfected in triplicate and each well was assayed in triplicate. For each condition tested, the luciferase activity was normalized to the activity produced from empty vector.

\section{Statistical analysis}

All the values are expressed as the mean \pm standard error of mean (SEM). Statistical differences between the two groups were determined using Student's $t$-test. The correlation of PHF8 immunoreactivity with patients' clinicopathological variables was analyzed by the $\chi^{2}$ test or Fisher's exact test. The Kaplan-Meier method was used to estimate overall survival. Survival differences according to PHF8 or miR-125b expression were analyzed by the log-rank test. Regression analysis was performed using GraphPad Prism $^{\circledR}$. $P$-values of $<0.05$ were considered statistically significant.

\section{Results \\ PHF8 is upregulated in human PCa tissues and cell lines}

To investigate the potential role of the histone demethylase PHF8 in human PCa, we first profiled the expression pattern of PHF8 in human normal prostate tissues and cancer tissues. We analyzed 19 normal tissues and $89 \mathrm{PCa}$ tissues and found that PHF8 was upregulated in human PCa tissues at both mRNA and protein levels (Figure 1A and B). Furthermore, we analyzed the expression of PHF8 in three human PCa cell lines (LNCaP, PC-3, and DU145) and normal prostate epithelial cell lines (PNT2 and BPH-1). The results showed that PHF8 mRNA levels were high in PCa cells compared to normal epithelial cells (Figure 1C). Taken together, these findings demonstrate that PHF8 is upregulated in human $\mathrm{PCa}$ and indicate the potential role of PHF8 in human PCa. 

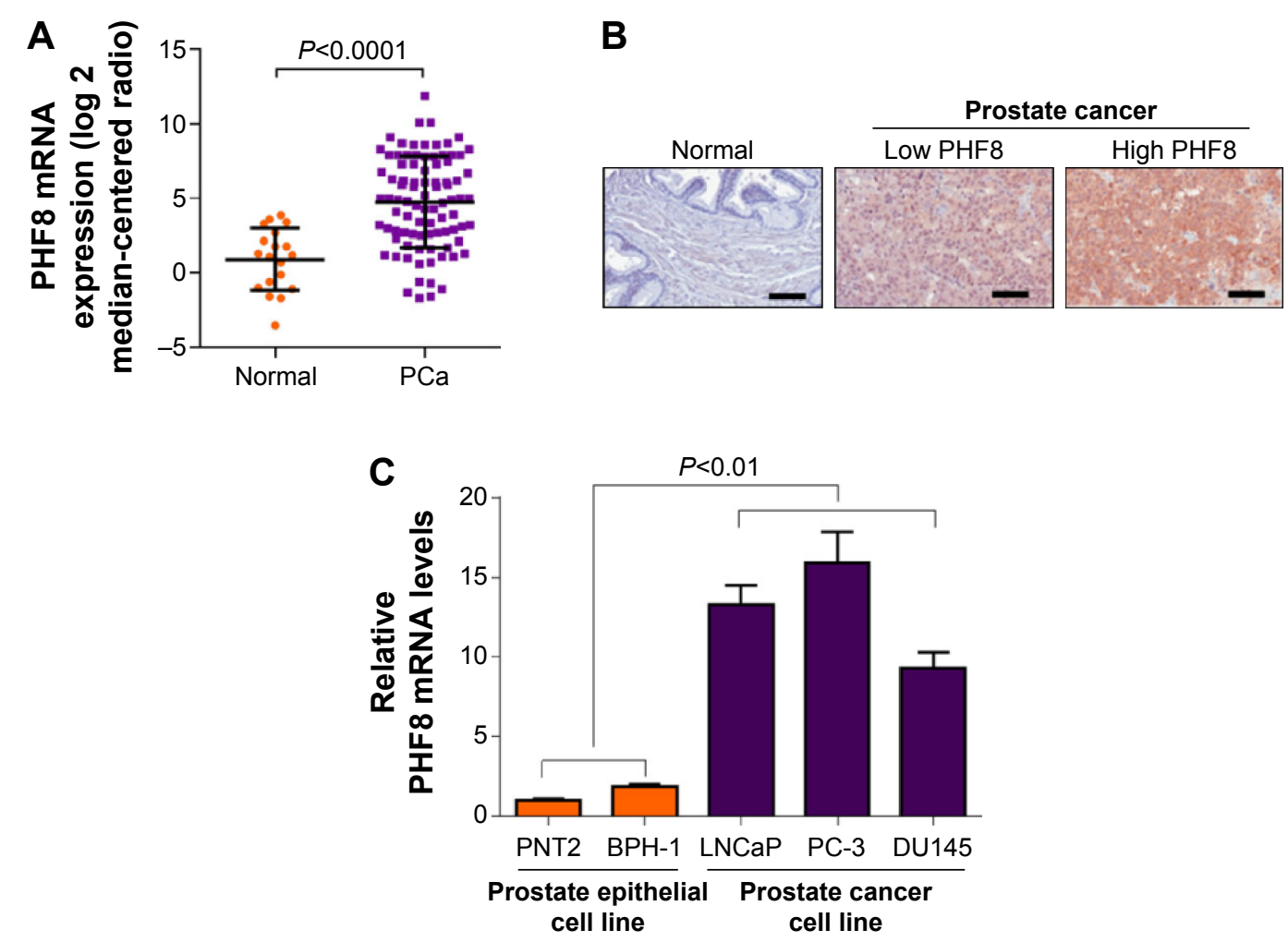

Figure I PHF8 is upregulated in human PCa and cell lines.

Notes: (A) PHF8 mRNA level is upregulated in human PCa. The mRNA level of PHF8 in adjacent normal tissues and cancer tissues from PCa patients was analyzed with q-PCR. $\mathrm{N}=19$ in normal group; $\mathrm{n}=89$ in PCa group. (B) Representative IHC results showing PHF8 protein level is upregulated in human PCa. Adjacent normal prostate tissues and PCa tissues were subjected to IHC analyses with anti-PHF8 antibody. Both low PHF8 expression and high PHF8 expression are shown. Scale is $50 \mu$ m. (C) PHF8 mRNA level is high in PCa cell lines. The mRNA level of PHF8 in normal prostate epithelial cells (PNT2 and BPH-I) and PCa cells (LNCaP, PC-3, and DUI45) was analyzed with q-PCR.

Abbreviations: PHF8, plant homeodomain finger protein 8; PCa, prostate cancer; IHC, immunohistochemical.

\section{PHF8 regulates PCa growth and cellular transformation}

Since our results indicated that PHF8 is involved in human $\mathrm{PCa}$, we explored whether PHF8 regulates the cellular behaviors of PCa cells. Therefore, we knocked down PHF8 in PCa cell lines, LNCaP, PC-3, and DU145. We found that $\mathrm{PHF} 8$ knockdown reduced cellular proliferation rate of $\mathrm{PCa}$ cells in vitro (Figure $2 \mathrm{~A}-\mathrm{C}$ ). We next probed the potential contribution of PHF8 to the transformative properties of PCa cells. We observed that PHF8-depleted cells possessed reduced colony-forming activity in LNCaP, PC-3, and DU145 cells (Figure 2D-F). Furthermore, we generated prostate cells with stably PHF8 knockdown and performed xenograft experiment. The results showed that PHF8 knockdown significantly inhibited the growth of PCa cells in vivo (Figure 2G-I). These evidences demonstrate that PHF8 promotes $\mathrm{PCa}$ cell growth and cellular transformation.

\section{PHF8 regulates apoptosis of PCa cells}

To investigate whether PHF8 regulates PCa cell proliferation and colony formation by orchestrating survival, we performed fluorescence-activated cell sorting to detect apoptotic cells. We found that PHF8 knockdown significantly increased the number of apoptotic cells in LNCaP, PC-3, and DU145 cells (Figure 3A). We next performed TUNEL assay to investigate whether DNA damage is involved in apoptosis induced by PHF8 knockdown. Markedly, PHF8 knockdown increased the number of TUNEL-positive cells in PCa cells, indicating that PHF8 knockdown induced DNA damage (Figure 3B). Finally, we performed Western blot to analyze the apoptotic signaling pathways. The results indicated that PHF8 knockdown activated apoptotic pathway and inactivated antiapoptotic pathway. In detail, PHF8 knockdown increased the protein level of Bax, p21, cleaved caspase 3, and cleaved PARP, while the antiapoptotic protein $\mathrm{Bcl}-2$ was downregulated when PHF8 was knocked down (Figures 3C and S1). Taken together, PHF8 regulates survival of human PCa cells.

\section{PHF8 promotes miR-I25b expression in human $\mathrm{PCa}$}

miR-125b is widely accepted as an oncogenic microRNA, and its role has been deeply investigated in human PCa. 
A
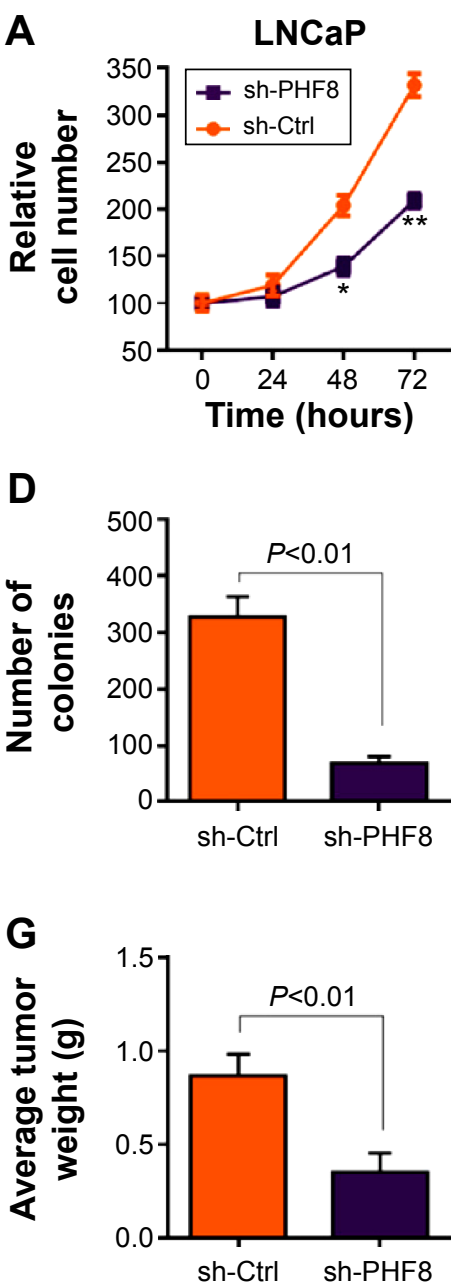
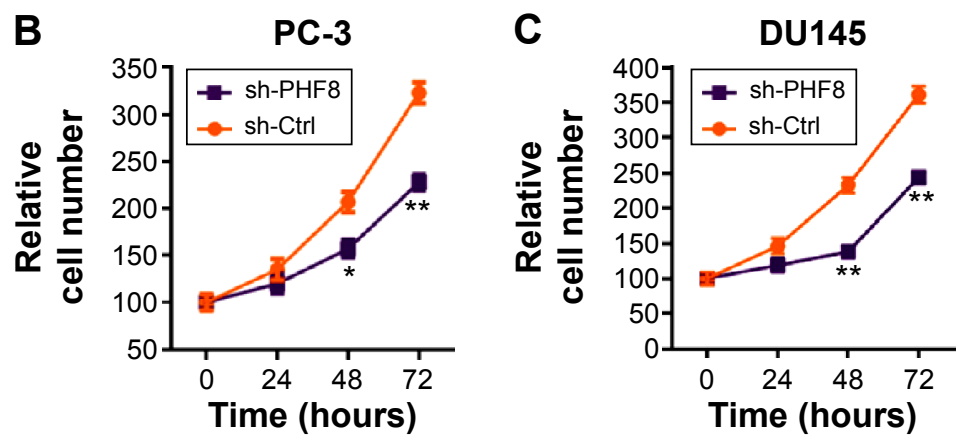

E

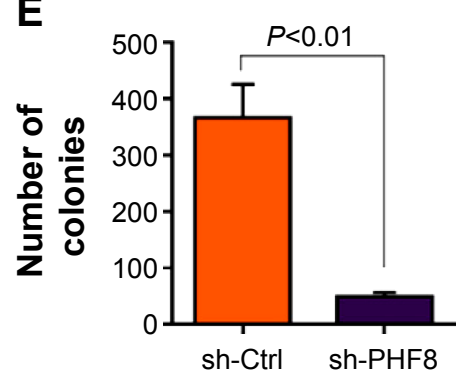

$\mathbf{F}$
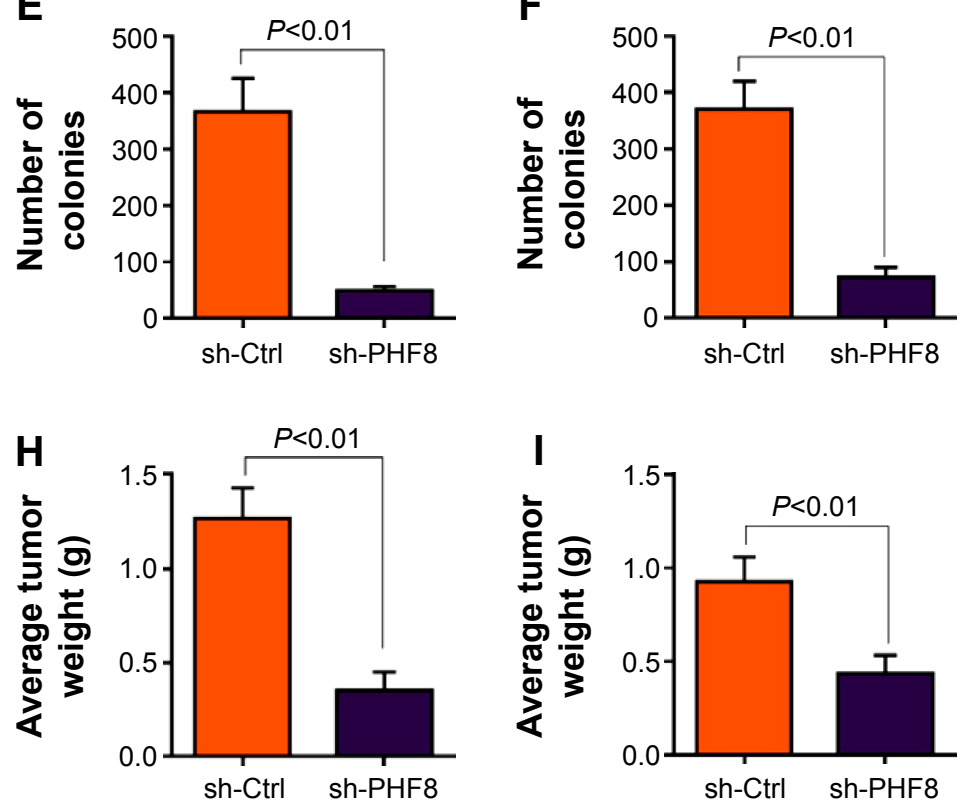

Figure 2 PHF8 knockdown inhibits prostate cancer cell growth in vitro and in vivo.

Notes: (A-C) PHF8 knockdown attenuates human prostate cancer cell proliferation. LNCaP (A), PC-3 (B), and DUI45 (C) cells were infected with retrovirus carrying sh-Ctrl or sh-PHF8, and the cell numbers were evaluated with MTT method at 24 hours, 48 hours, and 72 hours postinfection. $* P<0.05$, $* * P<0.01$ vs sh-Ctrl. (D-F) Control cells or those with sh-PHF8 transduction were subjected to soft sugar colony formation assay. The number of colonies was analyzed 2 weeks later. (D) LNCaP cells, (E) PC-3 cells, and (F) DUI45 cells were quantified. N=3 in each group. PHF8 knockdown represses in vivo growth of human prostate cancer cells. Control prostate cancer cells or those with sh-PHF8 transduction were subjected to xenograft mice experiments (G-I). Tumor weight was evaluated at the terminal of the experiments. (G) LNCaP cells, (H) PC-3 cells, and (I) DUI45 cells were quantified. $\mathrm{N}=10$ in each group.

Abbreviation: PHF8, plant homeodomain finger protein 8.

miR-125b was reported to be an important antiapoptotic factor and regulated drug resistance in human PCa. Our q-PCR results also showed that miR-125b was upregulated in human PCa tissues (Figure 4A), which was consistent with previous reports. ${ }^{13}$ Furthermore, we performed linear regression analysis to figure out whether miR-125b level is correlated with PHF8 expression in human PCa. The results indicated that miR-125b level was significantly and positively correlated with PHF8 level in human PCa tissues (Figure 4B), indicating that PHF8 may regulate miR-125b. We next knocked down PHF8 in LNCaP cells and found that PHF8 knockdown significantly reduced the level of miR-125b (Figure 4C). Finally, our luciferase assay demonstrated that PHF8 overexpression enhanced the promoter activity of miR-125b (Figure 4D). Taken together, these results demonstrate that $\mathrm{PHF} 8$ regulates miR-125b expression in human PCa.

\section{MiR-I25b is critically for PHF8 function in $\mathrm{PCa}$}

As we have demonstrated that PHF8 regressed the expression of miR-125b, we wanted to know whether miR-125b is essential for the function of PHF8 in human PCa. We knocked down miR-125b in LNCaP cells using specific LNA-antimiR-125b (Figure 5A). We found that miR-125b knockdown reduced $\mathrm{LNCaP}$ cell proliferation and induced apoptosis (Figure 5B and C). However, PHF8 knockdown 

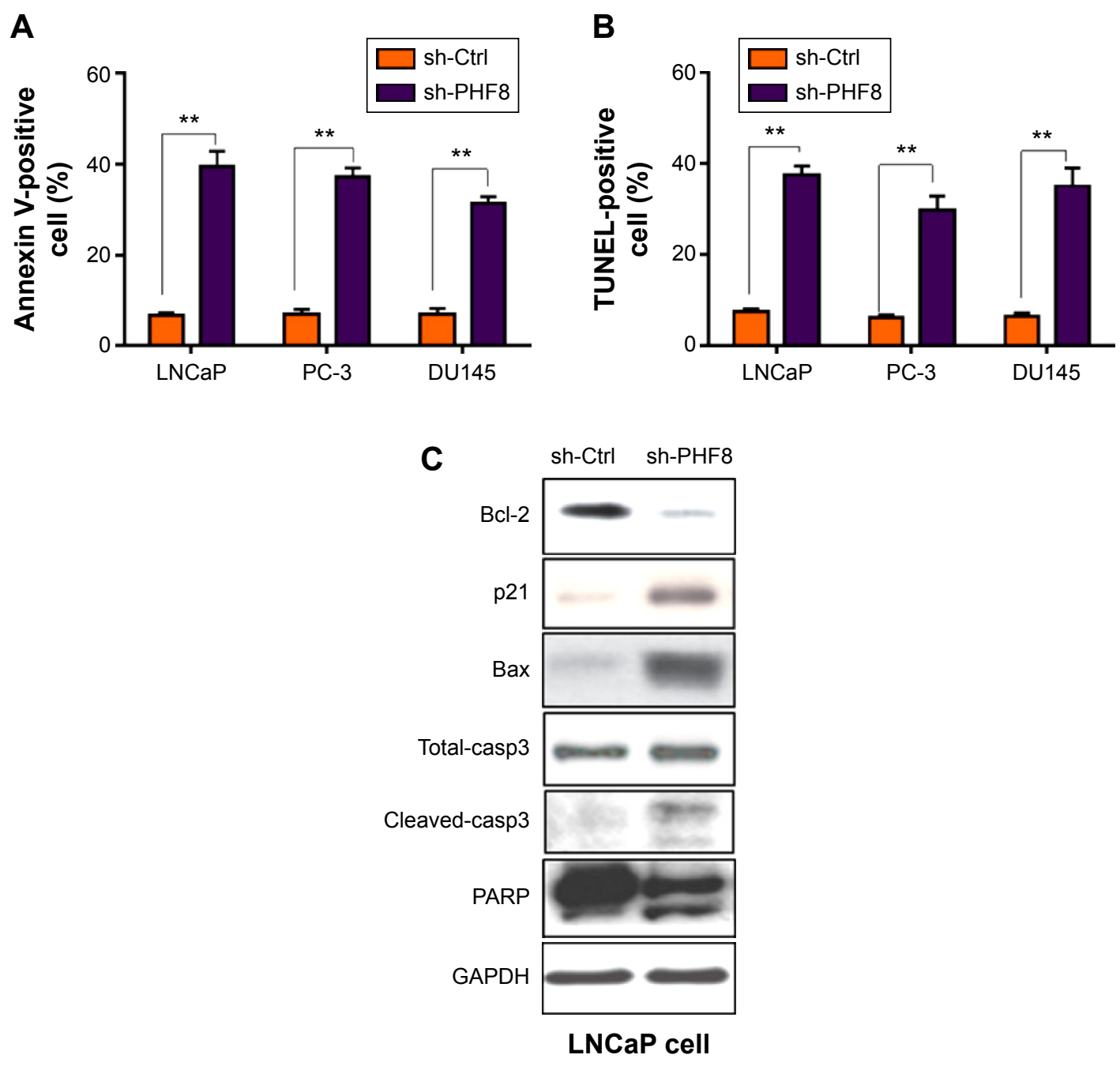

Figure 3 PHF8 regulates survival of prostate cancer cells.

Notes: (A) Prostate cancer cells were infected with retrovirus carrying sh-Ctrl or sh-PHF8 for 48 hours. Percentage of apoptotic cells was analyzed with FACS. PHF8 knockdown promotes apoptosis (Annexin V-positive) of LNCaP, PC-3, and DUI45 cells. **P $<0.01$. (B) PHF8 knockdown increases percentage of TUNEL-positive cells. Prostate cancer cells were infected with retrovirus carrying sh-Ctrl or sh-PHF8 for 48 hours, and then, TUNEL assay was performed to analyze DNA damage. (C) Representative Western blot showing PHF8 knockdown activates caspase 3, Bax, p2I, and PARP and inhibits Bcl-2 in LNCaP cells. LNCaP cells were infected with retrovirus carrying sh-Ctrl or sh-PHF8 for 48 hours, and then, Western blot was performed to analyze protein level.

Abbreviations: PHF8, plant homeodomain finger protein 8; FACS, fluorescence-activated cell sorting; TUNEL, transferase-mediated dUTP nick end labeling.

was unable to affect LNCaP cell proliferation and apoptosis when miR-125b was knocked down (Figure 5B and C). These results demonstrate that miR-125b is critically essential for the function of PHF8 in human PCa.

\section{Discussion}

PHF8 functions essentially in many developmental and disease processes. Mutations in PHF8 are associated with X-linked mental retardation and cleft lip/cleft palate. ${ }^{10,22-24}$ Histone demethylation modulated by PHF8 is suggested to play a critical role in neuronal differentiation, brain, and craniofacial development. ${ }^{4,25}$ Recently, PHF8 was reported to participate in several types of cancers, including leukemia, esophageal squamous cell carcinoma (ESCC), non-smallcell lung cancer, and PCa. In acute promyelocytic leukemia, PHF8 governs retinoic acid response. All-trans retinoic acid (ATRA) sensitivity depends on the enzymatic activity and phosphorylation status of PHF8, which in turn can be pharmacologically manipulated to resurrect ATRA sensitivity to resistant cells. ${ }^{26}$ Oncogenic features of PHF8 in ESCC were also reported. ${ }^{27}$ Currently, PHF8 was shown to regulate lung cancer cells growth and survival by targeting miR-21. ${ }^{28}$

Björkman et $\mathrm{al}^{29}$ carried out a systematic, genome-wide analysis of the functional significance of 615 epigenetic proteins in PCa cells. They identified that PHF8 was overexpressed in $\mathrm{PCa}$ with an impact on cell proliferation, migration, 

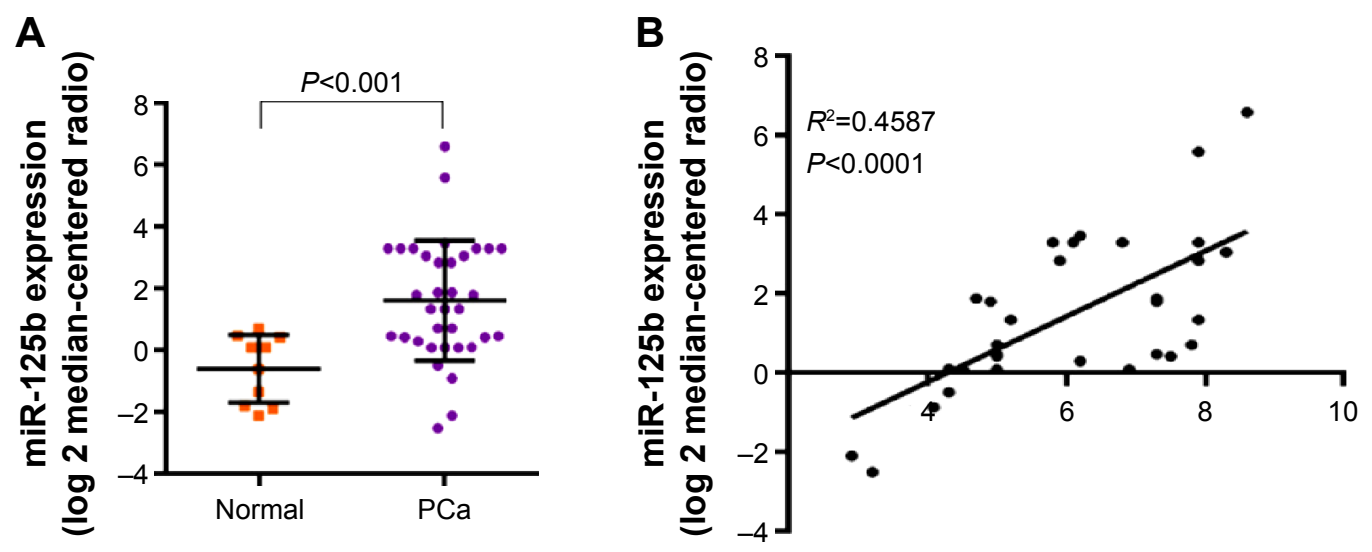

\section{PHF8 mRNA expression ( $\log 2$ median-centered radio)}
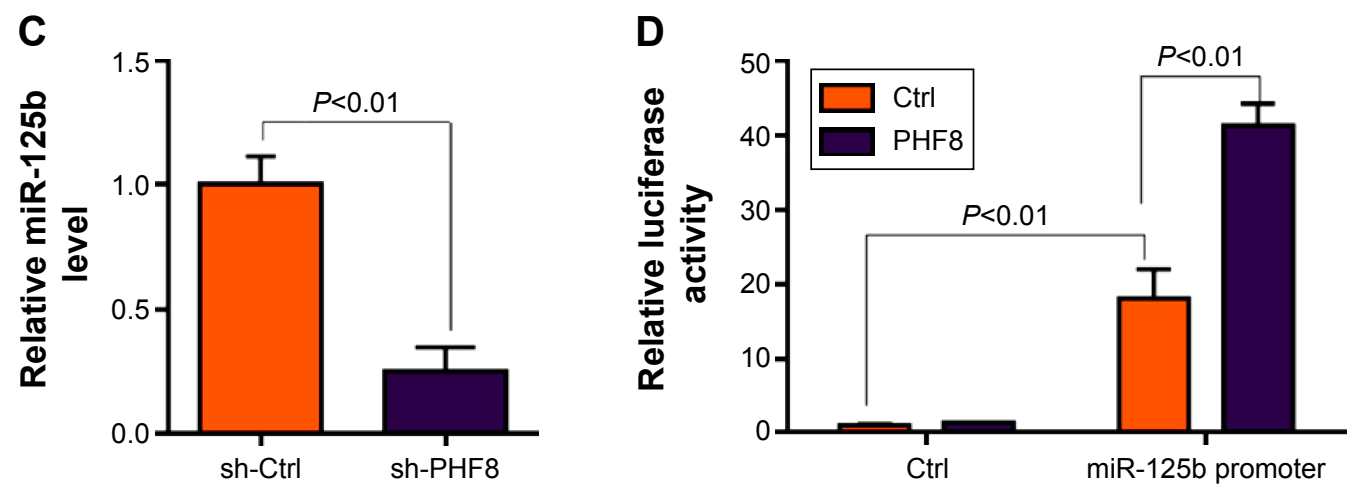

Figure 4 PHF8 regulates the expression of oncomiR miR-125b.

Notes: (A) miR-125b is overexpressed in human PCa tissues. The level of miR-125b in adjacent normal tissues and cancer tissues from PCa patients was analyzed with q-PCR. $\mathrm{N}=10$ in normal group; $\mathrm{n}=35$ in PCa group. (B) miR-125b expression level is correlated with PHF8 expression level. Linear regression analysis was performed to analyze the correlation between miR-125b level and PHF8 mRNA level. N=35. (C) PHF8 knockdown reduces miR-I25b expression level. (D) PHF8 promotes miR-I25b promoter activity. A pcDNA4.0 vector was used as the control of PHF8 expressing vector, and a pGL3-Basic vector without miR-125b promoter was used as a control luciferase vector.

Abbreviations: Ctrl, control; PHF8, plant homeodomain finger protein 8; PCa, prostate cancer.

and invasion. ${ }^{29}$ Inconsistent with the finding of Björkman et $\mathrm{al}^{29}$ we found that PHF8 was overexpressed in human PCa tissues and cell lines (Figure 1). In the present study, we also found that PHF8 knockdown inhibits cellular proliferation of PCa cells (LNCaP, PC-3, and DU145) in vitro and in vivo (Figure 2). Furthermore, cellular transformation of PCa cells was also reduced by PHF8 knockdown (Figure 2). In addition, we showed that PHF8 knockdown induces apoptosis in PCa cells by upregulating apoptotic protein (Bax, p-21, cleaved caspase 3, and cleaved PARP) and downregulating the antiapoptotic protein Bcl-2. Noticeably, DNA damage is involved in this effect (Figure 3). Together with the previous reports, our evidence indicates that PHF8 regulates cellular proliferative, transformative, and survival behaviors of cancer cells.

The oncomiR miR-125b has been reported to participate in human PCa. ${ }^{19}$ miR-125b was found to have the ability of rendering $\mathrm{LNCaP}$ cells resistant to androgen withdrawal.
It was found to be androgen regulated and one of its targets, BAK1, was identified as being involved in how these PCa cells undergo apoptosis functionally. ${ }^{30}$ miR-125b promotes growth of PCa xenograft tumor through targeting proapoptotic genes. ${ }^{18}$ Both p53-dependent and p53-independent pathways are involved in miR-125b-mediated apoptosis in PCa. ${ }^{16}$ For a long time, the underlying mechanism by which miR-125b is regulated remained unknown. We found that PHF8 could activate the promoter of miR-125b and promote its expression in human PCa cells (Figure 4). PHF8 knockdown induced apoptosis in PCa cells, and p53 may be involved in this process, because p21, a downstream target of p53 was upregulated when PHF8 was knocked down (Figure 3), which may be attributed to the downregulation of miR-125b. Finally, we demonstrated that miR-125b was critically essential for PHF8 effects on proliferation and apoptosis of PCa cells (Figure 5). 
A

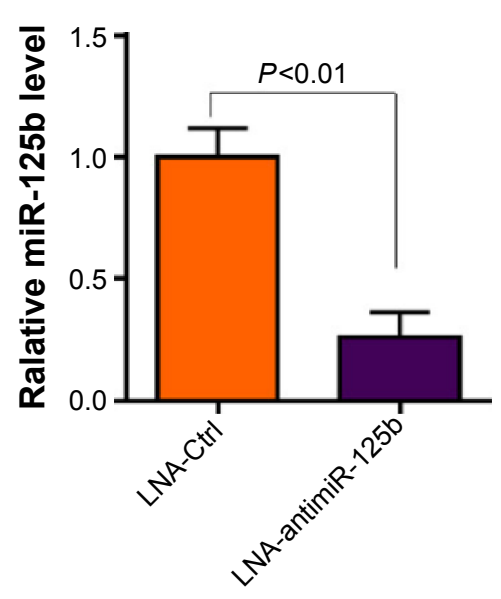

B

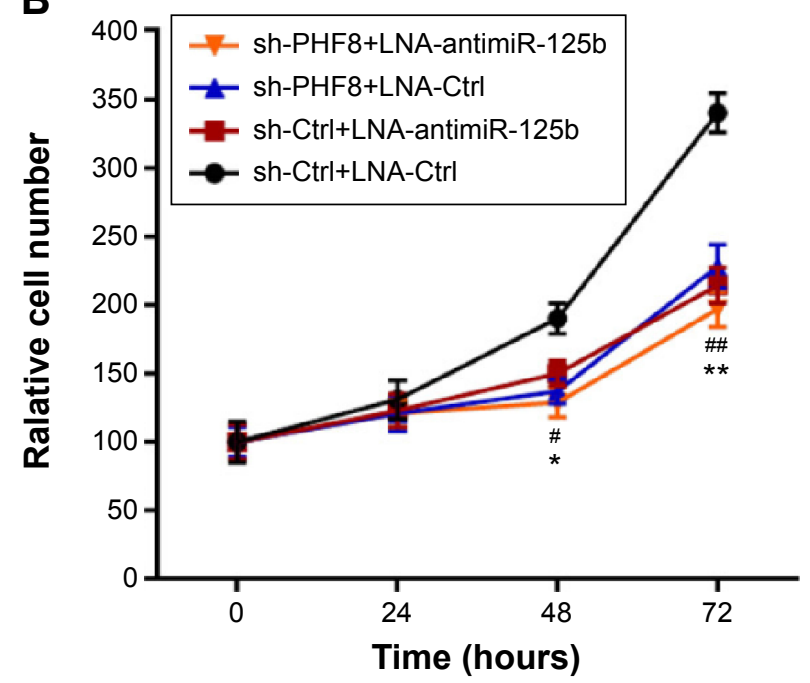

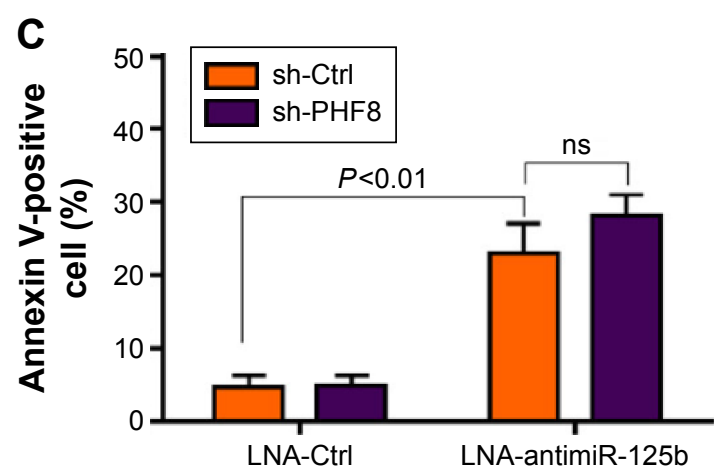

Figure 5 miR-125b knockdown is sufficient to block the effects of PHF8 in prostate cancer cells.

Notes: (A) miR-125b knockdown in LNCaP cells. LNCaP cells were transfected with LNA-Ctrl or LNA-antimiR-125b for 48 hours, and then, RNA was harvested and subjected to cDNA synthesis and q-PCR. (B) miR-125b knockdown blocks PHF8 effect on LNCaP cell proliferation. LNCaP cells were transfected with LNA-Ctrl or LNA-antimiR-125b for 24 hours, and then were infected with indicated retrovirus, and the cell number was evaluated with MTT method at 24 hours, 48 hours, and 72 hours postinfection. ${ }^{*} P<0.05,{ }^{\# P}<0.0$ l indicate sh-Ctrl+LNA-Ctrl vs sh-Ctrl+LNA-antimiR-I25b; $* P<0.05$, $* * P<0.01$ indicate sh-Ctrl+LNA-Ctrl vs sh-PHF8+LNA-Ctrl. (C) miR-I25b knockdown blocks PHF8 effect on LNCaP cell apoptosis. LNCaP cells were transfected with LNA-Ctrl or LNA-antimiR-125b for 24 hours, and then were infected with the indicated retrovirus for 48 hours, and the percentage of apoptotic cells was analyzed with FACS.

Abbreviations: PHF8, plant homeodomain finger protein 8; LNA, locked nucleic acid; ns, no significance; FACS, fluorescence-activated cell sorting.

\section{Conclusion}

In conclusion, we identify the histone demethylase PHF8 as an oncogenic protein in human $\mathrm{PCa}$. These findings indicate PHF8 as a potential candidate for clinical intervention.

\section{Disclosure}

The authors report no conflicts of interest in this work.

\section{References}

1. Jemal A, Murray T, Samuels A, Ghafoor A, Ward E, Thun MJ. Cancer statistics, 2003. CA Cancer J Clin. 2003;53:5-26.

2. Karan D, Lin MF, Johansson SL, Batra SK. Current status of the molecular genetics of human prostatic adenocarcinomas. Int J Cancer. 2003;103: 285-293.

3. Abate-Shen C, Shen MM. Molecular genetics of prostate cancer. Genes Dev. 2000;14:2410-2434.

4. Qi HH, Sarkissian M, Hu GQ, et al. Histone H4K20/H3K9 demethylase PHF8 regulates zebrafish brain and craniofacial development. Nature. 2010; 466:503-507.
5. Liu W, Tanasa B, Tyurina OV, et al. PHF8 mediates histone $\mathrm{H} 4$ lysine 20 demethylation events involved in cell cycle progression. Nature. 2010;466:508-512.

6. Feng W, Yonezawa M, Ye J, Jenuwein T, Grummt I. PHF8 activates transcription of rRNA genes through $\mathrm{H} 3 \mathrm{~K} 4 \mathrm{me} 3$ binding and $\mathrm{H} 3 \mathrm{~K} 9 \mathrm{me} 1 / 2$ demethylation. Nat Struct Mol Biol. 2010;17:445-450.

7. Lee MG, Norman J, Shilatifard A, Shiekhattar R. Physical and functional association of a trimethyl H3K4 demethylase and Ring6a/MBLR, a polycomb-like protein. Cell. 2007;128:877-887.

8. Pasini D, Hansen KH, Christensen J, Agger K, Cloos PA, Helin K. Coordinated regulation of transcriptional repression by the RBP2 H3K4 demethylase and polycomb-repressive complex 2. Genes Dev. 2008;22: $1345-1355$.

9. Zhu Z, Wang Y, Li X, et al. PHF8 is a histone H3K9me2 demethylase regulating rRNA synthesis. Cell Res. 2010;20:794-801.

10. Laumonnier F, Holbert S, Ronce N, et al. Mutations in PHF8 are associated with $\mathrm{X}$ linked mental retardation and cleft lip/cleft palate. $J$ Med Genet. 2005;42:780-786.

11. Lin HM, Castillo L, Mahon KL, et al. Circulating microRNAs are associated with docetaxel chemotherapy outcome in castration-resistant prostate cancer. Br J Cancer. 2014;110:2462-2471. 
12. Alshalalfa M, Bader GD, Goldenberg A, Morris Q, Alhajj R. Detecting microRNAs of high influence on protein functional interaction networks: a prostate cancer case study. BMC Syst Biol. 2012;6:112.

13. Sun D, Layer R, Mueller AC, et al. Regulation of several androgeninduced genes through the repression of the miR-99a/let-7c/miR125b-2 miRNA cluster in prostate cancer cells. Oncogene. 2014;33: 1448-1457.

14. Nguyen HC, Xie W, Yang M, et al. Expression differences of circulating microRNAs in metastatic castration resistant prostate cancer and low-risk, localized prostate cancer. Prostate. 2013;73:346-354.

15. Feng N, Xu B, Tao J, et al. A miR-125b binding site polymorphism in bone morphogenetic protein membrane receptor type IB gene and prostate cancer risk in China. Mol Biol Rep. 2012;39:369-373.

16. Amir S, Ma AH, Shi XB, Xue L, Kung HJ, Devere White RW. Oncomir miR-125b suppresses p14(ARF) to modulate p53-dependent and p53independent apoptosis in prostate cancer. PLoS One. 2013;8:e61064.

17. Giangreco AA, Vaishnav A, Wagner D, et al. Tumor suppressor microRNAs, miR-100 and-125b, are regulated by 1,25-dihydroxyvitamin D in primary prostate cells and in patient tissue. Cancer Prev Res (Phila). 2013;6: 483-494.

18. Shi XB, Xue L, Ma AH, Tepper CG, Kung HJ, White RW. miR-125b promotes growth of prostate cancer xenograft tumor through targeting pro-apoptotic genes. Prostate. 2011;71:538-549.

19. Lin KY, Zhang XJ, Feng DD, et al. miR-125b, a target of CDX2, regulates cell differentiation through repression of the core binding factor in hematopoietic malignancies. J Biol Chem. 2011;286:38253-38263.

20. Han W, Xin Z, Zhao Z, et al. RNA-binding protein PCBP2 modulates glioma growth by regulating FHL3. J Clin Invest. 2013;123: 2103-2118.

21. Kim JK, Noh JH, Jung KH, et al. Sirtuin7 oncogenic potential in human hepatocellular carcinoma and its regulation by the tumor suppressors MiR-125a-5p and MiR-125b. Hepatology. 2013;57:1055-1067.
22. Koivisto AM, Ala-Mello S, Lemmelä S, Komu HA, Rautio J, Järvelä I. Screening of mutations in the PHF8 gene and identification of a novel mutation in a Finnish family with XLMR and cleft lip/cleft palate. Clin Genet. 2007;72:145-149.

23. Loenarz C, Ge W, Coleman ML, et al. PHF8, a gene associated with cleft lip/palate and mental retardation, encodes for an Nepsilon-dimethyl lysine demethylase. Hum Mol Genet. 2010;19:217-222.

24. Kleine-Kohlbrecher D, Christensen J, Vandamme J, et al. A functional link between the histone demethylase PHF8 and the transcription factor ZNF711 in X-linked mental retardation. Mol Cell. 2010;38:165-178.

25. Qiu J, Shi G, Jia Y, et al. The X-linked mental retardation gene PHF8 is a histone demethylase involved in neuronal differentiation. Cell Res. 2010;20:908-918.

26. Arteaga MF, Mikesch JH, Qiu J, et al. The histone demethylase PHF8 governs retinoic acid response in acute promyelocytic leukemia. Cancer Cell. 2013;23:376-389.

27. Sun X, Qiu JJ, Zhu S, et al. Oncogenic features of PHF8 histone demethylase in esophageal squamous cell carcinoma. PLoS One. 2013; 8:e77353.

28. Shen Y, Pan X, Zhao H. The histone demethylase PHF8 is an oncogenic protein in human non-small cell lung cancer. Biochem Biophys Res Commun. 2014;451:119-125.

29. Björkman M, Östling P, Härmä V, et al. Systematic knockdown of epigenetic enzymes identifies a novel histone demethylase PHF8 overexpressed in prostate cancer with an impact on cell proliferation, migration and invasion. Oncogene. 2012;31:3444-3456.

30. DeVere White RW, Vinall RL, Tepper CG, Shi XB. MicroRNAs and their potential for translation in prostate cancer. Urol Oncol. 2009;27: $307-311$. 


\section{Supplementary materials}

Table SI Primers for qPCR

\begin{tabular}{lll}
\hline Name & Forward primer $\mathbf{( \mathbf { 5 } ^ { \prime } \mathbf { - 3 } \mathbf { \prime } )}$ & Reverse primer $\mathbf{( \mathbf { 5 } ^ { \prime } \mathbf { - 3 } \mathbf { \prime } )}$ \\
\hline PHF8 & GTGCCGGTGTATTGCCTCT & CAACACAACTGCCATGAAACC \\
GAPDH & CCACCCATGGCAAATTCCATGGCA & TCTAGACGGCAGGTCAGGTCCACC \\
miR-I25b & GCCCTCCCTGAGACCTCAA & GTGCAGGGTCCGAGGT \\
U6 & GCGCGTCGTGAAGCGTTC & GTGCAGGGTCCGAGGT \\
\hline
\end{tabular}

Abbreviation: PHF8, plant homeodomain finger protein 8.

Table S2 sh-RNA used to targeting PHF8

\begin{tabular}{ll}
\hline sh-RNA name & Sequences \\
\hline sh-PHF8 & GCTGGAGCAGTTCCTCTAATC \\
\hline
\end{tabular}

Abbreviation: PHF8, plant homeodomain finger protein 8.

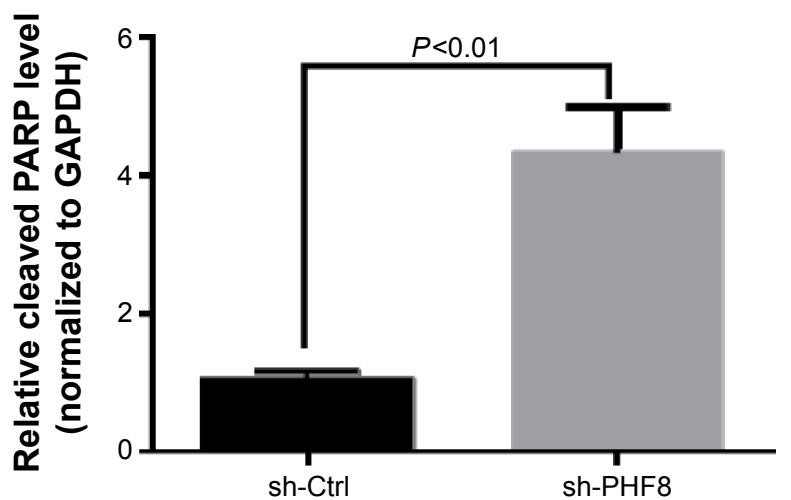

Figure SI Relative level of cleaved PARP related to Figure $3 \mathrm{C}$.

Note: The data are presented as mean \pm SEM of three independent experiments. Abbreviations: SEM, standard error of mean; PHF8, plant homeodomain finger protein 8.
OncoTargets and Therapy

\section{Publish your work in this journal}

OncoTargets and Therapy is an international, peer-reviewed, open access journal focusing on the pathological basis of all cancers, potential targets for therapy and treatment protocols employed to improve the management of cancer patients. The journal also focuses on the impact of management programs and new therapeutic agents and protocols on

\section{Dovepress}

patient perspectives such as quality of life, adherence and satisfaction. The manuscript management system is completely online and includes a very quick and fair peer-review system, which is all easy to use. Visit http://www.dovepress.com/testimonials.php to read real quotes from published authors. 